OPEN ACCESS

Edited by:

Avindra Nath

National Institute of Neurological Disorders and Stroke-NINDS (NIH),

United States

Reviewed by:

Kiran Teresa Thakur,

Columbia University Medical Center,

United States

Bryan Smith,

National Institutes of Health $(\mathrm{NIH})$,

United States

Kenichi Oishi,

Johns Hopkins University,

United States

*Correspondence:

Kristy O. Murray

kmurray@bcm.edu

Specialty section:

This article was submitted to

Neuroinfectious Diseases,

a section of the journal

Frontiers in Neurology

Received: 16 October 2017

Accepted: 14 February 2018

Published: 27 March 2018

Citation:

Murray KO, Nolan MS, Ronca SE, Datta S, Govindarajan K,

Narayana PA, Salazar L, Woods SP and Hasbun $R$ (2018) The

Neurocognitive and MRI Outcomes

of West Nile Virus Infection:

Preliminary Analysis Using an

External Control Group.

Front. Neurol. 9:111.

doi: 10.3389/fneur.2018.00111

\section{The Neurocognitive and MRI Outcomes of West Nile Virus Infection: Preliminary Analysis Using an External Control Group}

\author{
Kristy O. Murray ${ }^{1 *}$, Melissa S. Nolan ${ }^{1}$, Shannon E. Ronca ${ }^{1}$, Sushmita Datta ${ }^{2}$, \\ Koushik Govindarajan ${ }^{2}$, Ponnada A. Narayana ${ }^{2}$, Lucrecia Salazar ${ }^{2}$, Steven P. Woods ${ }^{3}$ \\ and Rodrigo Hasbun ${ }^{4}$
}

${ }^{1}$ Department of Pediatrics, Section of Pediatric Tropical Medicine, The National School of Tropical Medicine, Baylor College of Medicine, Texas Children's Hospital, Houston, TX, United States, ${ }^{2}$ Department of Diagnostic and Interventional Imaging, The University of Texas Health Science Center at Houston, Houston, TX, United States, ${ }^{3}$ Department of Psychology, University of Houston, Houston, TX, United States, ${ }^{4}$ Department of Internal Medicine, The University of Texas Health Science Center at Houston, Houston, $T X$, United States

To understand the long-term neurological outcomes resultant of West Nile virus (WNV) infection, participants from a previously established, prospective WNV cohort were invited to take part in a comprehensive neurologic and neurocognitive examination. Those with an abnormal exam finding were invited for MRI to evaluate cortical thinning and regional brain atrophy following infection. Correlations of presenting clinical syndrome with neurologic and neurocognitive dysfunctions were evaluated, as well as correlations of neurocognitive outcomes with MRI results. From 2002 to 2012, a total of 262 participants with a history of WNV infection were enrolled as research participants in a longitudinal cohort study, and 117 completed comprehensive neurologic and neurocognitive evaluations. Abnormal neurological exam findings were identified in 49\% (57/117) of participants, with most abnormalities being unilateral. The most common abnormalities included decreased strength (26\%; 30/117), abnormal reflexes (14\%; 16/117), and tremors (10\%; 12/117). Weakness and decreased reflexes were consistent with lower motor neuron damage in a significant proportion of patients. We observed a $22 \%$ overall rate of impairment on the Repeatable Battery for the Assessment of Neuropsychological Status (RBANS), with impairments observed in immediate (31\%) and delayed memory (25\%). On MRI, participants showed significant cortical thinning as compared to age- and gender-matched controls in both hemispheres, with affected regions primarily occurring in the frontal and limbic cortices. Regional atrophy occurred in the cerebellum, brain stem, thalamus, putamen, and globus pallidus. This study provides valuable new information regarding the neurological outcomes following WNV infection, with $\mathrm{MRI}$ evidence of significant cortical thinning and regional atrophy; however, it is important to note that the results may include systemic bias due to the external control group. Considering no effective treatment measures are available, strategies to prevent infection are key.

Keywords: West Nile virus, cortical thinning, regional brain atrophy, Repeatable Battery for the Assessment of Neuropsychological Status, neurocognitive outcomes, neurological outcomes 


\section{INTRODUCTION}

With West Nile virus (WNV) now endemic throughout North America, with upwards of three million infected cases, it is a priority to document the long-term clinical outcomes (1-3). Among a large cohort of participants with a history of WNV infection in Houston, TX, USA, we found that $40 \%$ of those who presented with clinical disease continued to experience WNV-related morbidity up to 8 years postinfection (4). This percentage was up to $80 \%$ for those who initially presented with encephalitis. Sequelae varied in severity, with a higher than expected proportion developing chronic neurological and renal disease (5-10). Other shorter-term studies report neurocognitive impairment, debilitating fatigue, persistent neuromuscular paralysis, tremors, and depression as outcomes experienced by patients with a history of West Nile Neuroinvasive Disease (WNND) $(7,11-16)$. There are currently no published studies correlating neurocognitive evaluations to neuroimaging outcomes in patients with WNND.

MRI is the most sensitive neuroimaging technique for intracranial infections because of its superior soft tissue detail, sensitivity to subtle inflammatory processes, vascular imaging techniques, and functional imaging. Limited MRI data are available to evaluate the long-term effects of WNV, and published studies of brief case report describe changes during acute infection, with areas of interest including the basal ganglia, thalamus, pons, and lobar gray and white matter (17-19). The goal of this study was to understand the long-term neurological function impairment, neurocognitive deficits, and/ or MRI abnormal findings in patients with a history of WNV infection.

\section{MATERIALS AND METHODS}

\section{Study Population}

Following the first index WNV case in Houston, TX, USA in 2002, a cohort of WNV patients was established in collaboration with the City of Houston Health Department, Harris County Public Health, and the Gulf Coast Regional Blood Center. From 2002 to 2012 , a total of 262 patients were enrolled as research participants in this longitudinal cohort. For the current study, 72 enrolled participants were unavailable due to death $(n=29)$, additional tests determined a false positive WNV status $(n=11)$, lost-tofollow-up since initial enrollment $(n=26)$, or self-withdrew from further participation since initial enrollment $(n=6)$. Of the 190 invited participants, 117 (62\%) agreed to take part in the neurologic and the neurocognitive evaluations (Figure 1), and 30 took part in the MRI study (Figure 1). Failure to take part $(n=73)$ was due to inability to come to the Texas Medical Center for extensive testing, lack of desire to participate, or unspecified reasons.

\section{Standard Protocol Approvals, Registrations, and Patient Consents}

This study was approved by Institutional Review Boards at both Baylor College of Medicine (H-30533) and the University of

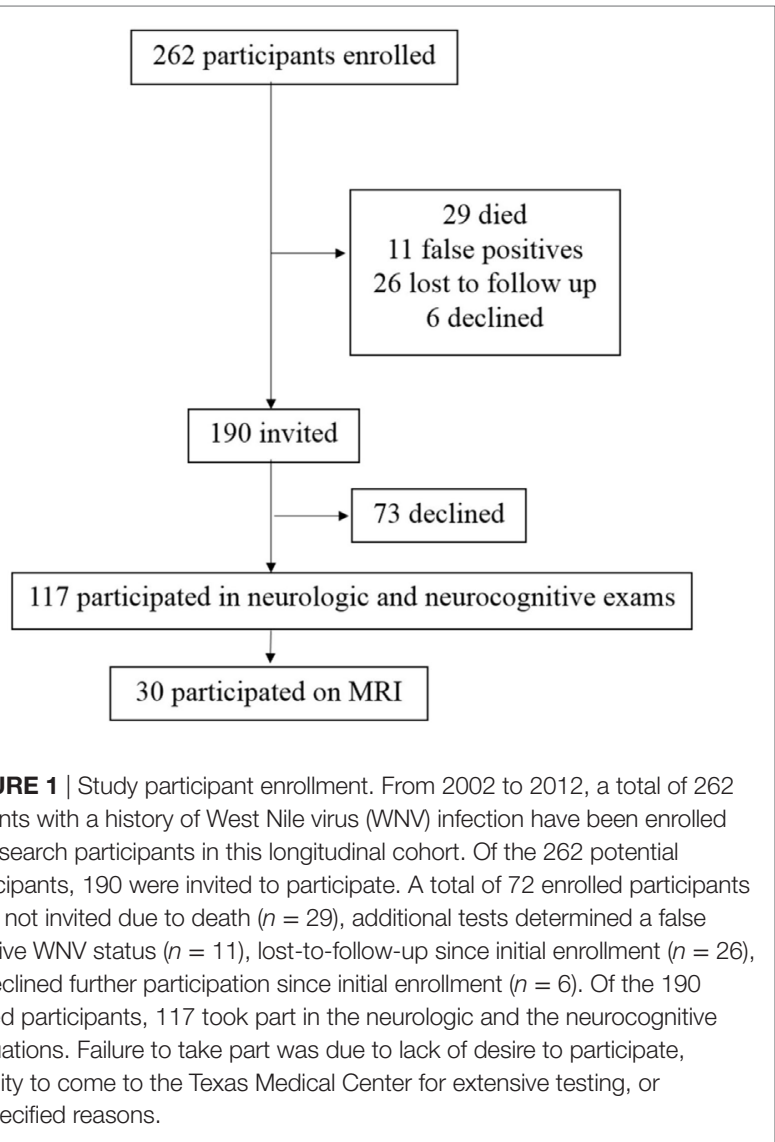

Texas Health Science Center at Houston (HSC-SPH-03-039). All participants provided informed written consent for their involvement in the study. Participants were registered as part of study protocol at the Memorial Hermann's Clinical Innovation and Research Institute and University of Texas 3T MRI Center in Houston, TX, USA.

\section{Neurologic Examinations}

To evaluate the sensory and motor aspects of infection, we used the collaborative antiviral study group neurological exam case report form utilized in the WNV immunoglobulin trial (20). Two physicians evaluated cranial nerves, deep tendon reflexes, fine touch sensory sensations, muscle strength, resting tremors, cerebellar dysmetria, and tone. Physical abilities were measured using the Barthel Index. Mental status was evaluated using the Glasgow Coma Scale and Glasgow Outcome Score $(21,22)$.

\section{Neurocognitive Examinations}

To evaluate the psychological aspects of sequelae, neurocognitive evaluations were performed. These included multiple validated questionnaires and task-based exams to assess various aspects of cognitive functions. These evaluations were performed at Memorial Hermann's Clinical Innovation and Research Institute, Houston, TX, USA. Quality of life and depression were measured using the Short Form-36 Health Survey and Becks Depression 
Index-II (BDI), respectively (23). The Weschler Abbreviated Scale of Intelligence (WASI) Matrix Reasoning subtest evaluated perceptual reasoning. Gesture to command tested apraxia (difficulty of motor planning to perform a task). The token test assessed aphasia (language impairment). The Galveston Orientation and Amnesia test (GOAT) measured attention and orientation (24). Finally, we administered the Repeatable Battery for the Assessment of Neuropsychological Status (RBANS), which provides index scores measuring immediate memory, visuospatial/constructional, language, attention and delayed memory (23).

\section{MRI}

Participants with an abnormal neurological exam, neurological complaints, and/or and RBANS score less than 85 were invited to participate in the MRI studies. MRI studies were completed at the University of Texas at Houston MRI Facility using a 3T Philips Ingenia MRI scanner. The MRI protocol included multiple sequences. In this study, the analysis was based on MPRAGE 3D T1-weighted images. These images were acquired in the sagittal plane with an isotropic resolution of $1 \mathrm{~mm}$. The other imaging parameters were: $\mathrm{TR}=8.1 \mathrm{~ms}, \mathrm{TE}=3.7 \mathrm{~ms}$, FOV $=256 \mathrm{~mm} \times 256 \mathrm{~mm}$, number of slices $=181$. Thirty subjects consisting of 18 females and 12 males with an average age of 46 years $( \pm 12)$ were compared to 30 scans on age- and gendermatched normal controls selected from the OASIS database ${ }^{1}$ for comparing regional atrophy and cortical thickness in the WNV subjects.

\section{Cortical Thickness Analysis}

Cortical thickness analysis was carried out on the FreeSurfer ${ }^{2}$ software pipeline (version 5.1) $(25,26)$. The pipeline consists of a volumetric stream and a surface-based stream. Initially, all the volumes were registered to template space and then underwent intensity normalization. After that, a skull-stripping procedure based on a combination of a watershed algorithm and deformable template model was applied to extract the brain from the images. Following this, the output brain mask was labeled using a probabilistic atlas where each voxel in the normalized brain mask volume is assigned one of the following labels: cerebral white matter, cerebral cortex, lateral ventricle, inferior lateral ventricle, cerebellum white matter, cerebellum cortex, thalamus, caudate, putamen, pallidum, hippocampus, amygdala, accumbens area, third ventricle, fourth ventricle, brainstem, and cerebrospinal fluid (27).

The surface-based stream consists of a white matter segmentation followed by tessellation that identifies gray matter and white matter boundary and pial surfaces $(25,27,28)$. Likely, white matter points were identified using voxel intensity and neighborhood intensity information. White and pial surfaces were constructed after refining from initial surfaces generated for each hemisphere. The thickness is defined as the average of

${ }^{1}$ https://www.oasis-brains.org/.

${ }^{2}$ http://surfer.nmr.mgh.harvard.edu/fswiki. the distance between the surface and the gray and white matter (GM-WM) boundary and the distance between the GM-WM boundary and the surface (28).

FreeSurfer implements a technique for automatically assigning a neuroanatomical label to each location on a cortical surface. It uses both geometric information derived from the cortical model and neuroanatomical convention to form a complete labeling of the cortical gyri and sulci. FreeSurfer uses the Desikan-Killiany Atlas and the Destrieux Atlas. For this study, the labels based on Desikan-Killiany atlas were used for cortical thickness measurements, and maps were generated for both the participant and control datasets.

\section{Regional Atrophy Analysis}

3D T1-weighted images underwent preprocessing that included skull-stripping and intensity non-uniformities correction. Nonlinear symmetric diffeomorphic registration technique $(29,30)$ was applied to co-align all the pre-processed 3D T1 images to International Consortium for Brain Mapping (ICBM) template. ${ }^{3}$ For each subject, a 3D diffeomorphic map was generated during non-linear registration which was used to calculate Jacobian determinant at each voxel (31).

\section{Statistical Analyses}

Descriptive statistics were performed to describe the outcome variables of the cross-sectional study on prospectively followed participants. Univariate analyses were then conducted with the Pearson's X2 test or Fisher's exact test to identify factors of neurological and neurocognitive exams that were significant between clinical descriptions of WNV cases. For cortical thickness, FreeSurfer's inbuilt generalized linear model (GLM) was used to determine group differences between WNV cases and controls. Using the GLM, uncorrected group difference maps for cortical thickness were generated. These maps represent voxel-wise differences in cortical thickness between the WNV cases and selected controls. Following this, a multiple comparison Monte-Carlo simulation was performed with a voxel-wise threshold of $p=0.05$ and a cluster-wise threshold of $p=0.05$, using 5,000 iterations. The resulting output maps represent the significant regions of differences between the two groups.

For regional atrophy studies, atrophy of any region on $3 \mathrm{D}$ $\mathrm{T} 1$ in comparison to that of ICBM template were determined by the positive and negative values of average JDs of the region. Logarithmic of JDs at each voxels within intracranial brain were calculated following the normalization of JDs that accounts for the brain size. These values at each voxel were obtained for both participants and healthy controls. Healthy control data were obtained as described above. Group analysis of the average normalized logarithmic JDs between participants and controls for each voxel was done using statistical tools available in Statistical Parametric Mapping. The voxels with significant differences were obtained based on the false discovery rate of 0.05 and were considered as the voxels representing atrophy.

${ }^{3}$ http://www.loni.usc.edu/ICBM/Downloads/Downloads_ICBMtemplate.shtml. 
We were also interested in analyzing the association between neurocognitive deficits and cortical thickness. To reduce Type I error, we examined pairwise correlations between the RBANS total score and the cortical thickness regions of interest using Pearson product moment coefficients. Given the exploratory nature of these analyses, we set the critical alpha level at 0.01 .

TABLE 1 | Baseline demographics of 117 adults with history of West Nile virus infection who underwent a complete neurological examination. ${ }^{\text {a }}$

\begin{tabular}{|c|c|}
\hline Baseline variables & $N=117(\%)$ \\
\hline Mean age at exam (range) & 57 years $(18-89)$ \\
\hline Male gender & $63(53)$ \\
\hline \multicolumn{2}{|l|}{ Race/ethnicity } \\
\hline White, non-Hispanic & $98(83)$ \\
\hline White, Hispanic & $10(8)$ \\
\hline Black & $6(6)$ \\
\hline Asian & $3(3)$ \\
\hline \multicolumn{2}{|c|}{ Acute clinical presentation at time of West Nile virus (WNV) infection } \\
\hline Encephalitis & $35(30)$ \\
\hline Meningitis & $16(14)$ \\
\hline Uncomplicated fever & $39(33)$ \\
\hline Asymptomatic & $27(23)$ \\
\hline \multicolumn{2}{|l|}{ Education level } \\
\hline High school degree & $42(36)$ \\
\hline Undergraduate degree & $40(34)$ \\
\hline Graduate degree & $33(28)$ \\
\hline Less than high school degree & $3(2)$ \\
\hline \multicolumn{2}{|l|}{ Employment status at time of exam } \\
\hline Full-time employment & $55(47)$ \\
\hline Retired & $38(32)$ \\
\hline Unemployed, disability, or other employment & $22(19)$ \\
\hline Part-time employment & $3(2)$ \\
\hline \multicolumn{2}{|l|}{ Comorbid conditions } \\
\hline Hypertension & $38(32)$ \\
\hline Diabetes & $17(15)$ \\
\hline \multicolumn{2}{|l|}{ Time between WNV infection and neurologic exam } \\
\hline 0-3 years & $26(22)$ \\
\hline $4-7$ years & $46(39)$ \\
\hline $8-11$ years & $45(38)$ \\
\hline
\end{tabular}

${ }^{a}$ Neurologic exam included evaluation of cranial nerves, deep tendon reflexes, fine touch sensory sensations, muscle strength, resting tremors, cerebellar dysmetria, and tone.

\section{RESULTS}

Table 1 shows the demographics of our study population, which consisted of mostly white, non-Hispanic (83\%) individuals, and an almost equal proportion of males (53\%) and females. Mean age at the time of exam was 57 years (range 18-89 years). Most participants had graduated high school (98\%), and almost half (47\%) were employed full time at the time of their neurological exam. Almost one-third of participants were hypertensive (32\%), and $15 \%$ were diabetic. The majority of participants (77\%) were four or more years past their acute WNV infection. Participants evaluated had a history of asymptomatic disease (23\%), West Nile Fever (WNF) (33\%), or WNND (44\%).

Across the entire cohort, almost half $(49 \% ; 57 / 117)$ of participants had some type of abnormal neurological exam finding, with most abnormalities being unilateral in nature (Tables 2 and $\mathbf{3}$ ). The most common abnormalities included abnormal (decreased) strength $(26 \% ; 30 / 117)$, abnormal reflexes $(14 \% ; 16 / 117)$, and tremors $(10 \% ; 12 / 117)$. Decreased strength was more common among core muscle groups than in the extremities, with the exception of finger strength, and was associated with decreased reflexes in 16 out of 30 patients consistent with lower motor neuron involvement. Abnormal reflexes were consistent among all five muscle groups. Finally, tremors were more common among the upper body than the lower body, which was distinct from other findings. There was no statistical difference between time from infection ( 5 years or more vs. less than 5 years) and having an abnormal neurological examination $(p=0.73)$.

The frequencies of gross neurocognitive and ADL abnormalities were quite low, with fewer than $5 \%$ of the cohort producing abnormal scores on the GCS, GOS, GOAT, and Barthel Index (Tables 4 and 5). Similarly, fewer than 5\% of the sample had IQ scores in the Borderline or extremely low range (i.e., <80). However, the frequency of milder higher-order neurocognitive and mood disturbances was somewhat higher. Using a 1SD cutpoint derived from published normative standards, we observed a $22 \%$ overall rate of impairment on the RBANS, with the domains of immediate (31\%) and delayed memory (25\%) being the most frequently impaired. Seven percent of participants had RBANS total scores in the moderately to severely impaired range

TABLE 2 | Neurological exam findings in 117 adults with West Nile virus infection by acute clinical picture. ${ }^{2}$

\begin{tabular}{|c|c|c|c|c|c|c|c|}
\hline \multirow[b]{2}{*}{ Area of examination } & \multirow[b]{2}{*}{$\begin{array}{l}\text { All participants } \\
\qquad n=117\end{array}$} & \multicolumn{2}{|c|}{ Non-neuroinvasive } & \multicolumn{2}{|c|}{ Neuroinvasive $^{a}$} & \multirow[b]{2}{*}{$\begin{array}{c}\text { Odds ratio }{ }^{\mathrm{b}} \text { (95\% } \\
\text { confidence interval) }\end{array}$} & \multirow[b]{2}{*}{$p$-Value } \\
\hline & & $\begin{array}{c}\text { No symptoms } \\
n=27\end{array}$ & $\begin{array}{l}\text { West Nile fever } \\
\qquad n=39\end{array}$ & $\begin{array}{c}\text { Meningitis } \\
n=16\end{array}$ & $\begin{array}{c}\text { Encephalitis } \\
\qquad n=35\end{array}$ & & \\
\hline Abnormal neurological & 57 & 7 & 20 & 11 & 19 & $1.8(0.8-3.8)$ & $N^{c}$ \\
\hline Abnormal cranial nerve & 8 & 4 & 4 & 0 & 0 & Undefined & NS \\
\hline Abnormal reflexes ${ }^{d}$ & 16 & 0 & 4 & 3 & 9 & $4.2(1.2-14.6)$ & 0.02 \\
\hline Abnormal sensory & 8 & 0 & 3 & 4 & 1 & $1.9(0.4-8.8)$ & NS \\
\hline Abnormal strength & 30 & 1 & 10 & 4 & 12 & $2.5(1.1-6.3)$ & 0.04 \\
\hline Tremors & 12 & 2 & 3 & 1 & 6 & $1.5(0.4-5.4)$ & NS \\
\hline Abnormal cerebellare & 2 & 0 & 1 & 0 & 1 & 0.7 (0.03-13.9) & NS \\
\hline Abnormal tone & 1 & 0 & 1 & 0 & 0 & Undefined & NS \\
\hline
\end{tabular}

Meningitis, encephalitis, or acute flaccid paralysis presentation.

${ }^{b}$ Adjusted for age, gender, and history of diabetes mellitus.

cNon-significant $p$-value of $>0.05$.

${ }^{\mathrm{A}}$ All reflexes were decreased.

eCerebellar ataxia. 
TABLE 3 | Neurological exam findings on 117 adults with West Nile virus infection.

\begin{tabular}{|c|c|c|c|}
\hline Area of examination & $\begin{array}{c}\text { Total number } \\
\text { with } \\
\text { abnormal } \\
\text { findings (\%) }\end{array}$ & $\begin{array}{l}\text { Number with } \\
\text { abnormal } \\
\text { findings on } \\
\text { one side of the } \\
\text { body }(\%)\end{array}$ & $\begin{array}{l}\text { Number with } \\
\text { abnormal } \\
\text { findings on both } \\
\text { sides of the } \\
\text { body (\%) }\end{array}$ \\
\hline \multicolumn{4}{|l|}{ Cranial nerves } \\
\hline Ptosis & $3 / 117(3)$ & $0 / 117$ & $3 / 117$ \\
\hline Visual field deficit & $5 / 116(4)$ & $1 / 116$ & $4 / 116$ \\
\hline Extraocular movements & $0 / 117(0)$ & $0 / 117$ & $0 / 117$ \\
\hline Facial palsy & 0/117 (0) & $0 / 117$ & $0 / 117$ \\
\hline \multicolumn{4}{|c|}{ Abnormal reflexes (decreased) } \\
\hline Biceps & 10/117 (9) & $1 / 117$ & $9 / 117$ \\
\hline Brachioradialis & $10 / 117(9)$ & $1 / 117$ & $9 / 117$ \\
\hline Triceps & $12 / 117(10)$ & $0 / 117$ & $12 / 117$ \\
\hline Quadriceps & 9/116 (8) & $3 / 116$ & $6 / 116$ \\
\hline Gastrocnemius & $5 / 116(4)$ & $1 / 116$ & $4 / 116$ \\
\hline \multicolumn{4}{|l|}{ Abnormal sensation } \\
\hline Upper extremities & 3/117 (3) & $2 / 117$ & $1 / 117$ \\
\hline Lower extremities & $5 / 115(4)$ & $1 / 115$ & $4 / 115$ \\
\hline Face & $0 / 117(0)$ & $0 / 117$ & $0 / 117$ \\
\hline \multicolumn{4}{|l|}{ Decreased strength } \\
\hline Deltoid & $7 / 117(6)$ & $1 / 117$ & $6 / 117$ \\
\hline Biceps & $6 / 117(5)$ & $1 / 117$ & $5 / 117$ \\
\hline Brachioradialis & $5 / 117(4)$ & $0 / 117$ & $5 / 117$ \\
\hline Triceps & $8 / 117(7)$ & $3 / 117$ & $5 / 117$ \\
\hline Iliopsoas & 6/115 (5) & $1 / 115$ & $5 / 115$ \\
\hline Quadriceps & $7 / 116(6)$ & $2 / 116$ & $5 / 116$ \\
\hline Hamstrings & $5 / 116(4)$ & $0 / 116$ & $5 / 116$ \\
\hline Grip & $7 / 117(6)$ & $3 / 117$ & $4 / 117$ \\
\hline Foot dorsiflexion & $5 / 115(4)$ & $2 / 115$ & $3 / 115$ \\
\hline Foot plantar flexion & $2 / 114(2)$ & $0 / 114$ & $2 / 114$ \\
\hline Toe wiggling & $1 / 114(0.9)$ & $0 / 114$ & $1 / 114$ \\
\hline Arm drifts & 3/117 (3) & $2 / 117$ & $1 / 117$ \\
\hline $\begin{array}{l}\text { Firmly hold paper } \\
\text { between third and } \\
\text { fourth fingers }\end{array}$ & $17 / 117(15)$ & $5 / 117$ & $12 / 117$ \\
\hline \multicolumn{4}{|l|}{ Tremors } \\
\hline Face & 2/117 (2) & $1 / 117$ & $1 / 117$ \\
\hline Upper extremity & $11 / 117(9)$ & $4 / 117$ & $7 / 117$ \\
\hline Lower extremity & 0/117 (0) & $0 / 117$ & $0 / 117$ \\
\hline \multicolumn{4}{|l|}{ Abnormal cerebellar exam } \\
\hline Finger to nose & $2 / 117(2)$ & $0 / 117$ & $2 / 117$ \\
\hline Heel to shin & $1 / 116(0.9)$ & $0 / 116$ & $1 / 116$ \\
\hline
\end{tabular}

$(<2 \mathrm{SD})$. There was no significant difference between timing of the exam (five or more years since infection versus less than 5 years) and low RBANS score $(p=0.16)$. Consistent with previous findings, approximately one-fifth of the sample reported current depressed mood using a conservative BDI cutoff score of 17 , which is commonly recommended in medical and neurological populations (32).

On MRI, WNV participants showed significant cortical thinning as compared to age- and gender-matched controls (Figure 2A; raw data in Tables S1 and S2 in Supplementary Material). Significant cortical thinning in the left hemisphere (LH) included parts of the posterior cingulate cortex (pink), parts of the superior frontal cortex and medial-orbito frontal region (magenta), anterior cingulate cortex and inferior frontal cortex (violet blue), parts of the cuneus (orange) and parahippocampal region (pale green), and in the right hemisphere
(RH) included parts of the middle and inferior temporal cortex, and supramarginal region (light blue), inferior frontal region and insular cortex (bright green and red), parts of the superior frontal cortex (light green, blue, and violet), cingulate cortex (light purple), and inferior frontal region (blue). Lower neurocognitive functioning as determined by total RBANS score was associated with cortical thinning in regions of the LH only: caudal middle frontal gyrus $(r=0.49, p=0.008)$, rostral middle frontal gyrus $(r=0.49, p=0.007)$, and supramarginal gyrus $(r=0.51$, $p=0.004)$. Scatterplots comparing cortical thickness and total RBAN score by region and hemisphere among WNV participants can be found in Figure 3.

Tensor-based morphometry analysis of participants' scans vs. control scans revealed significant regional atrophy in the overall cohort of $30 \mathrm{WNV}$ participants that underwent MRI (Figure 2B). Significant regional atrophy was identified in the cerebellum, brain stem, thalamus, putamen, and globus pallidus. No significant correlation between a low RBANS score and these regions was identified.

\section{DISCUSSION}

This study contributes one of the largest cross-sectional examinations of the neurological and neurocognitive outcomes post-WNV infection, and the only known study to link these quantitative measures to MRI findings, allowing for valuable insight into the pathology of infection. These findings can be useful in the clinical management of patients with a history of WNV, particularly those with WNND.

Results of the neurological exam showed weakness and decreased reflexes consistent with lower motor neuron damage in a substantial proportion of patients (Tables 2 and 3 ) and identified WNND participants to be at an increased risk for these outcomes, findings consistent with current literature. Weatherhead et al. described abnormal reflexes and muscle weakness as common abnormalities in a prospective study that evaluated WNND and WNF participants in the Houston WNV cohort 1-3 and 8-11 years after their initial WNV infection (10), with results indicating that participants with a history of WNND with encephalitis were more likely to have persistent and progressive abnormalities.

When comparing the results of the neurocognitive exams between neuroinvasive and non-neuroinvasive cases, we see a significant difference in only a few of the measures (GOAT, RBANS total score, RBANS immediate memory, RBANS visuospatial, and Barthel ADL Index) (Table 4). A larger than expected number of the WNF cases $(20 \%)$ had observable deficits. In order to meet the case definition of WNND, it was required to have a lumbar puncture procedure with evidence of pleocytosis; therefore, it is possible that these cases were misclassified as WNF, when indeed, they had WNND. Newer guidelines now permit the diagnosis of encephalitis without lumbar puncture if patients present with altered mental status for greater than $24 \mathrm{~h}$ with no alternative explanation and meet at least three of the following criteria: fever $38 \mathrm{C}$ or above, generalized or partial seizures unrelated to a preexisting seizure disorder, new onset of focal neurologic findings, abnormality of brain parenchyma on neuroimaging, 
TABLE 4 | Neuropsychological exam findings in adults with West Nile virus infection by acute clinical presentation.

\begin{tabular}{|c|c|c|c|c|}
\hline Domain & Non-neuro $n=76(\%)$ & Neuroinvasive $n=35(\%)$ & Univariate $p$-value & Multivariate $^{\mathrm{a}} \boldsymbol{p}$-values \\
\hline \multicolumn{5}{|l|}{ Neurological Screening } \\
\hline Glasgow Coma Scale & $15.0(0.0)$ & $15.0(0.0)$ & NS & - \\
\hline Glasgow Outcome Scale & $5.0(0.0)$ & $4.9(0.0)$ & NS & - \\
\hline GOAT & $96.9(0.4)$ & $94.8(0.6)$ & 0.004 & 0.030 \\
\hline \multicolumn{5}{|l|}{ Neurocognitive } \\
\hline WASI matrix reasoning & $52.5(1.2)$ & $50.7(1.8)$ & NS & - \\
\hline RBANS total & $100.2(2.0)$ & $91.0(3.1)$ & 0.013 & 0.060 \\
\hline Immediate memory & $94.3(2.0)$ & $86.8(3.2)$ & 0.050 & NS \\
\hline Delayed memory & $97.6(2.0)$ & $91.1(3.1)$ & NS & - \\
\hline Visuospatial & $108.0(1.8)$ & $98.2(2.8)$ & 0.004 & 0.011 \\
\hline Language & $96.9(1.2)$ & $93.5(1.9)$ & NS & - \\
\hline Attention & $104.1(2.3)$ & $99.9(3.5)$ & NS & - \\
\hline Token test & $33.9(0.4)$ & $33.6(0.6)$ & NS & - \\
\hline \multicolumn{5}{|l|}{ Mood } \\
\hline Beck depression inventory & $8.9(1.2)$ & $10.0(1.8)$ & NS & - \\
\hline \multicolumn{5}{|l|}{ Functioning } \\
\hline Barthel ADL Index & $100.0(0.6)$ & $95.9(0.9)$ & $<0.001$ & 0.004 \\
\hline Short Form-36 & $99.4(1.2)$ & $96.3(1.8)$ & NS & - \\
\hline
\end{tabular}

GOAT, Galveston Orientation and Amnesia Test; RBANS, Repeatable Battery for the Assessment of Neuropsychological Status; WASI, Wechsler Abbreviated Scale of Intelligence. ${ }^{a}$ Analyses controlled for age and education.

TABLE 5 | Neuropsychological exam findings in adults with West Nile virus infection.

\begin{tabular}{lccc}
\hline Domain & Mean (SD) & Range & $\begin{array}{c}\text { Clinically } \\
\text { abnormal (\%) }\end{array}$ \\
\hline Neurological screening & & & \\
$\quad$ Glasgow Coma Scale $(n=117)$ & $15.0(0.0)$ & $15-15$ & $0.0^{\mathrm{a}}$ \\
Glasgow Outcome Scale $(n=117)$ & $5.0(0.2)$ & $4-5$ & $3.4^{\mathrm{b}}$ \\
GOAT $(n=116)$ & $96.2(3.6)$ & $75-100$ & $0.8^{\mathrm{c}}$ \\
Neurocognitive & & & \\
WASI matrix reasoning $(n=110)$ & $51.3(10.9)$ & $20-76$ & $3.6^{\mathrm{d}}$ \\
RBANS total $(n=112)$ & $97.0(17.7)$ & $54-148$ & $22.3^{\mathrm{e}}$ \\
Immediate memory & $91.6(18.2)$ & $40-136$ & $31.3^{\mathrm{e}}$ \\
Delayed memory & $95.3(17.9)$ & $44-127$ & $25.0^{\mathrm{e}}$ \\
Visuospatial/constructional & $104.5(16.5)$ & $60-131$ & $13.4^{\mathrm{e}}$ \\
Language & $95.3(11.0)$ & $57-120$ & $9.8^{\mathrm{e}}$ \\
Attention & $102.9(19.4)$ & $53-150$ & $17.9^{\mathrm{e}}$ \\
Token test $(n=112)$ & $33.6(3.3)$ & $21-36$ & - \\
Mood & & & $22.4^{\mathrm{f}}$ \\
Beck Depression Inventory & $9.4(10.3)$ & $0-43$ & - \\
( $n=116)$ & & & \\
Functioning and quality of life & & & \\
Barthel ADL Index $(n=117)$ & $98.7(5.3)$ & $60-100$ & $2.6^{\mathrm{g}}$ \\
Short form-36 $(n=115)$ & $98.4(10.5)$ & $50-126$ & - \\
\hline
\end{tabular}

GOAT, Galveston Orientation and Amnesia Test; RBANS, Repeatable Battery for the Assessment of Neuropsychological Status; WASI, Wechsler Abbreviated Scale of Intelligence.

${ }^{a} \mathrm{GCS}<13$.

${ }^{b} \mathrm{GOS}<5$.

${ }^{\circ} \mathrm{GOAT}<76$.

${ }^{a}$ WASI T scores $<30$.

${ }^{e}$ RBANS standard scores $<84$.

${ }^{t} \mathrm{BD}$ l raw scores $\geq 17$.

'Barthel scores $<90$.

abnormality in electroencephalography (33). Since we do not have this information for all participants, we cannot reevaluate their classification in this study. We acknowledge this limitation and that the significance of WNND as a risk factor for sequelae may be underrepresented in this study population. Also, none of our patients evaluated had WNV-associated acute flaccid paralysis, which is known to have poorer clinical outcomes than other presentations of WNND $(5,6)$. It would be valuable in future studies to further distinguish neurological and neurocognitive outcomes among the different clinical presentations of WNND (meningitis vs. encephalitis vs. AFP), since these different presentations have varying severity of morbidity (4).

MRIs to evaluate cortical thinning and atrophy were performed only on participants with ongoing neurological complaints and/or a low RBANS score. When compared to age- and gender-matched controls from the OASIS database, cortical thinning was identified in the frontal and limbic lobes of both the left and RHs, with small regions of the temporal and parietal lobes also indicated in the RH. Interestingly, participants with a low RBANS score also had significant thinning within regions of the LH. Significant regions were within the middle frontal gyrus and supramarginal gyrus, both within the parietal lobe. The parietal lobe is responsible for sensation and perception and integrating sensory input, areas that were identified to be weakened in some participants. The lack of overlap between regions affected by WNV compared to controls and cortical thinning correlates of the RBANS analysis of WNV participants may be a simple artifact of measurement. Namely, the RBANS is a brief neurocognitive screening measure and does not include tasks that assay the frontal regions identified in the cortical thinning analysis. This lack of overlap warrants additional neurocognitive measures in future studies with larger participant groups.

Although most WNV-infected participants were recruited in the Houston area, we were not able to match controls from OASIS based on location for this study. Additionally, the OASIS database contains data from controls scanned with a different machine with regards to both field strength and manufacturer when compared to our scans (1.5T Siemens and 3T Philips, respectively). While these differences could impact our findings and lead to systematic 

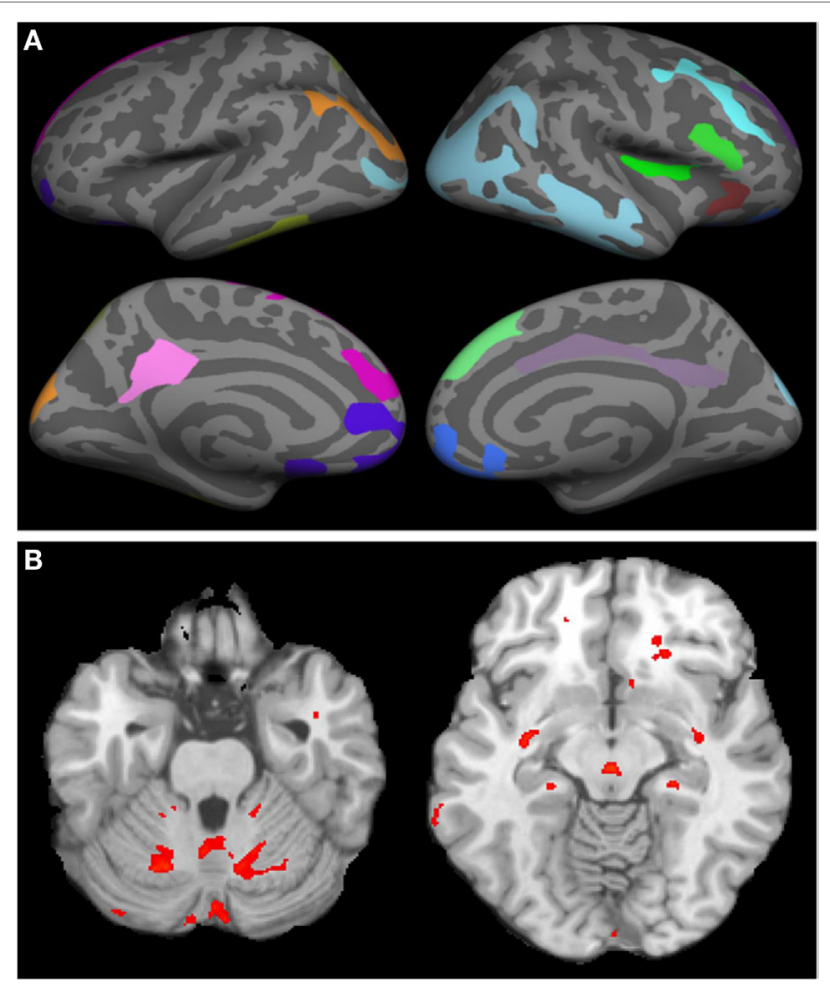

FIGURE 2 | Cortical thinning and regional atrophy analyses. (A) Volumetric MRI. Left (first column) and right (second column) hemispheres showing cortical thinning in West Nile infected individuals as compared to a database of MRIs from healthy controls with lateral (top row) and medial (bottom row) views. Colored regions indicate areas of thinning. In the left hemisphere, parts of the posterior cingulate cortex (pink), parts of the superior frontal cortex and medial-orbito frontal region (magenta), anterior cingulate cortex and inferior frontal cortex (violet blue), parts of the cuneus (orange) and para hippocampal region (pale green) were identified. In the right hemisphere, parts of the middle and inferior temporal cortex, and supramarginal region (light blue), inferior frontal region and insular cortex (bright green and red), parts of the superior frontal cortex (light green, blue, and violet), cingulate cortex (light purple), and inferior frontal region (blue) were identified. (B) Tensor-based morphometry (TBM) analysis of regional atrophy. Regions with regional atrophy as detected by TBM are identified in red.

bias, a recent publication from our group (34) found that when examining the effect of field strength between 1.5 and 3T, the cortical thickness showed field dependence only in certain regions that include posterior cingulate and parahippocampal regions in the $\mathrm{LH}$ and middle temporal cortex in the RH. In this current study on WNV, we observed significant cortical thinning in the LH that included parts of the posterior cingulate cortex (pink), parts of the superior frontal cortex and medial-orbito frontal region, anterior cingulate cortex and inferior frontal cortex, parts of the cuneus and para-hippocampal region, and in the $\mathrm{RH}$ included parts of the middle and inferior temporal cortex, and supramarginal region, inferior frontal region and insular cortex, parts of the superior frontal cortex (light green, blue, and violet), cingulate cortex, and inferior frontal region. Thus, many regions in which cortical thickness differences were observed in WNV patients did not show field dependence. Nonetheless, predicting systematic bias when using different scanners is difficult and this is an important limitation of our study. It will be crucial to confirm these results in future studies by concurrently enrolling and imaging control participants using the same scanners.

Regional atrophy was noted in the cerebellum, brain stem, thalamus, putamen, and globus pallidus of WNND patients who were not apparent in controls. These regions clearly mediate functions that are altered in WNND patients. Cerebellitis has only been described in one case report of a 10-year-old child (35). Infectious cerebellitis is most commonly caused by other etiologies such as JC virus, varicella zoster virus, and Listeria monocytogenes (36). The most common causes of brain stem/ rhomboencephalitis are L. monocytogenes and Enterovirus 71 (37). WNV can occasionally have brain stem involvement (38). Encephalitis with thalamic and basal ganglia involvement should raise the possibility of respiratory viruses and $\mathrm{WNV}$ infection $(39,40)$. This thalamic and basal ganglia involvement is responsible for the facial and upper extremity tremors, motor weakness, and abnormal reflexes seen in WNND (41). Although regional atrophy did not statistically correlate with the RBANS total score, atrophy in WNV participants compared to controls still provides pathological insights into the impact of WNND disease. Larger sample sizes within WNND with encephalitis, meningitis, and/ or acute flaccid paralysis would allow for further analysis of the effects of WNND and its specific clinical syndromes on regional atrophy. Also, serial MRI scans from acute disease onset to later timepoints would allow for documentation of atrophy and cortical thinning over time. Unfortunately, we were limited in this study to make comparisons to prior MRIs, as we only had MRI result data on six patients from the acute phase of illness, all of whom had a "normal" result provided.

Understanding the long-term complications of WNV infection are important to improving clinical outcomes and decreasing the costs on our health systems. A 2014 study determined that it can cost up to $\$ 400,000$ per patient to treat WNV sequelae, not including the cost of care during the acute phase of illness (42). This study provides new insights of long-term sequelae by comparing neurological, neuropsychological, and MRI results of affected patients 3-8 years after initial infection. Although some correlations of functional evaluations and MRI were not statistically significant, the biological relevance of the alterations identified via MRI are interesting. For the first time, this study identified brain areas of interest, such as the cerebellum, putamen, thalamus, supramarginal gyrus, and middle frontal gyrus, in patients continuing to experience neurological complications. These data will be crucial for future studies regarding the progression of WNV disease and associated long-term complications. Afflicted regions also suggest the damage pattern could be similar to what is seen in traumatic brain injury. At this time, with no specific preventive or therapeutic options to treat WNV infection other than supportive care, continuing these types of large-scale and long-term prospective studies will allow us to generalize expected consequences of infection. In turn, this allows for proactive treatment of sequelae that would otherwise lower a patients' quality of life. Ultimately, public health efforts to prevent infection are critical, as many of the issues identified in study participants are not reversible. 


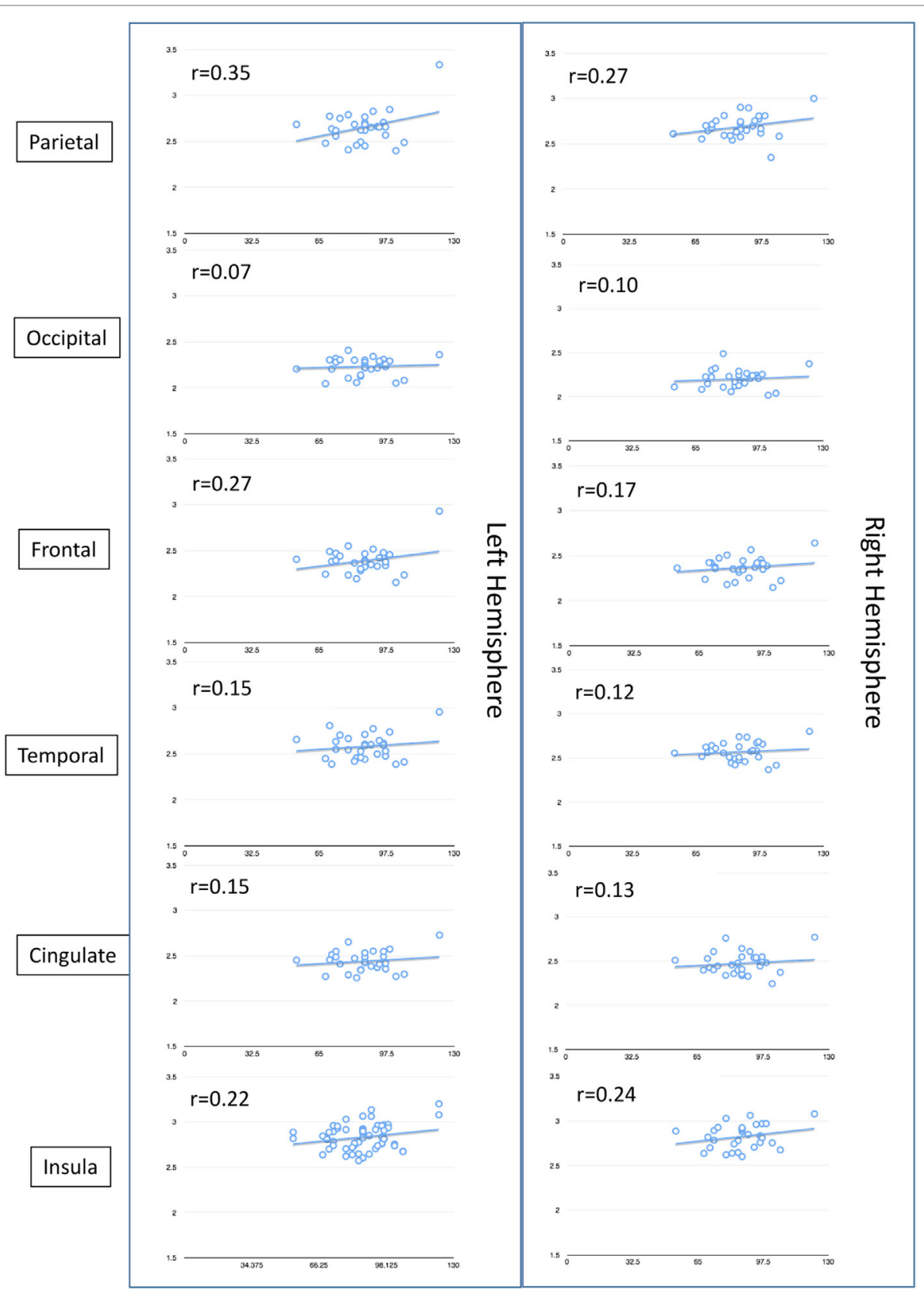

FIGURE 3 | Non-pairwise scatterplots of raw data compairing total Repeatable Battery for the Assessment of Neuropsychological Status score ( $y$-axis) to cortical thickness ( $x$-axis) by region and hemisphere of the brain among the 30 West Nile virus participants who underwent MRI.

\section{ETHICS STATEMENT}

This study was approved by Institutional Review Boards at both Baylor College of Medicine (H-30533) and the University of Texas Health Science Center at Houston (HSC-SPH-03-039). All participants provided informed written consent for their involvement in the study. Participants were registered as part of study protocol at the Memorial Hermann's Clinical Innovation and Research Institute and University of Texas 3T MRI Center in Houston, TX, USA.

\section{AUTHOR CONTRIBUTIONS}

Conception and design of study: KM, PN, SW, and RH. Acquisition and analysis of data: KM, MN, SR, SD, KG, LS, SW, and RH. Manuscript/figure drafting: KM, MN, SR, KG, PN, SW, and RH.

\section{ACKNOWLEDGMENTS}

The authors would like to thank the study participants and the clinical care staff at the Memorial Hermann Clinical Trials Unit, 
as well as Diana Martinez, Salma Khuwaja, Susan N. Rossman, and Raouf Arafat for their help founding the original Houston West Nile virus cohort.

\section{FUNDING}

This study was funded by NIH, National Institute for Allergy and Infectious Diseases (5R01AI091816-01, K23AI057341, and U19AI089992-01). The funders had no role in study design, data collection and analysis, decision to publish, or preparation

\section{REFERENCES}

1. Christou L. The global burden of bacterial and viral zoonotic infections. Clin Microbiol Infect. France: Christou, Clinical Microbiology and Infection 2011 European Society of Clinical Microbiology and Infectious Diseases (2011). p. 326-30.

2. Prow NA, Hewlett EK, Faddy HM, Coiacetto F, Wang W, Cox T, et al. The Australian public is still vulnerable to emerging virulent strains of West Nile virus. Front Public Health (2014) 2:146. doi:10.3389/fpubh.2014.00146

3. Benjelloun A, El Harrak M, Belkadi B. West Nile disease epidemiology in North-West Africa: bibliographical review. Transbound Emerg Dis (2015) 63(6):e153-9. doi:10.1111/tbed.12341

4. Murray KO, Garcia MN, Rahbar MH, Martinez D, Khuwaja SA, Arafat RR, et al. Survival analysis, long-term outcomes, and percentage of recovery up to 8 years post-infection among the Houston West Nile virus cohort. PLoS One (2014) 9:e102953. doi:10.1371/journal.pone.0102953

5. Sejvar JJ, Haddad MB, Tierney BC, Campbell GL, Marfin AA, Van Gerpen JA, et al. Neurologic manifestations and outcome of West Nile virus infection. JAMA (2003) 290:511-5. doi:10.1001/jama.290.4.511

6. Sejvar JJ, Bode AV, Marfin AA, Campbell GL, Pape J, Biggerstaff BJ, et al. West Nile virus-associated flaccid paralysis outcome. Emerg Infect Dis (2006) 12:514-6. doi:10.3201/eid1203.050643

7. Sejvar JJ, Curns AT, Welburg L, Jones JF, Lundgren LM, Capuron L, et al. Neurocognitive and functional outcomes in persons recovering from West Nile virus illness. J Neuropsychol (2008) 2:477-99. doi:10.1348/1748664 $07 \mathrm{X} 218312$

8. Murray K, Walker C, Herrington E, Lewis JA, Mccormick J, Beasley DW, et al. Persistent infection with West Nile virus years after initial infection. J Infect Dis (2010) 201:2-4. doi:10.1086/648731

9. Nolan MS, Podoll AS, Hause AM, Akers KM, Finkel KW, Murray KO. Prevalence of chronic kidney disease and progression of disease over time among patients enrolled in the Houston West Nile virus cohort. PLoS One (2012) 7:e40374. doi:10.1371/journal.pone.0040374

10. Weatherhead JE, Miller VE, Garcia MN, Hasbun R, Salazar L, Dimachkie MM, et al. Long-term neurological outcomes in West Nile virus-infected patients: an observational study. Am J Trop Med Hyg (2015) 92:1006-12. doi:10.4269/ ajtmh.14-0616

11. Murray KO, Resnick M, Miller V. Depression after infection with West Nile virus. Emerg Infect Dis (2007) 13:479-81. doi:10.3201/eid1303.060602

12. Berg PJ, Smallfield S, Svien L. An investigation of depression and fatigue post West Nile virus infection. S D Med (2010) 63:127-9, 131-23.

13. Gibney KB, Lanciotti RS, Sejvar JJ, Nugent CT, Linnen JM, Delorey MJ, et al. West Nile virus RNA not detected in urine of 40 people tested 6 years after acute West Nile virus disease. J Infect Dis (2011) 203:344-7. doi:10.1093/ infdis/jiq057

14. Leis AA, Stokic DS. Neuromuscular manifestations of West Nile virus infection. Front Neurol (2012) 3:37. doi:10.3389/fneur.2012.00037

15. Nolan MS, Hause AM, Murray KO. Findings of long-term depression up to 8 years post infection from West Nile virus. J Clin Psychol (2012) 68:801-8. doi: $10.1002 / j \mathrm{jclp} .21871$

16. Garcia MN, Hause AM, Walker CM, Orange JS, Hasbun R, Murray KO. Evaluation of prolonged fatigue post-West Nile virus infection and association of fatigue with elevated antiviral and proinflammatory cytokines. Viral Immunol (2014) 27:327-33. doi:10.1089/vim.2014.0035 of the manuscript. The MRI Center is a core facility for the NIH funded CCTS (grant \#UL1 TR000371). The purchase of MRI scanner was partially supported by NIH (grant \# 1 S10 RR19186-01).

\section{SUPPLEMENTARY MATERIAL}

The Supplementary Material for this article can be found online at http://www.frontiersin.org/articles/10.3389/fneur.2018.00111/ full\#supplementary-material.

17. Puchalski A, Liu A. A case of West Nile encephalitis that develops into a disease of deep white matter on MRI over a two-week span. Case Rep Neurol Med (2016) 2016:4389691. doi:10.1155/2016/4389691

18. Patel CB, Trikamji B, Mathisen G, Yim C, Zipser B, Mishra S. MRI ventral nerve root enhancement in five patients presenting with extremity weakness secondary to neuroinvasive West Nile virus. J Clin Neuromuscul Dis (2016) 1:41-3. doi:10.1097/01.cnd.0000496973.95654.fd

19. Ali M, Safriel Y, Sohi J, Llave A, Weathers S. West Nile virus infection: MR imaging findings in the nervous system. AJNR Am J Neuroradiol (2005) 26(2):289-97.

20. Hart J Jr, Tillman G, Kraut MA, Chiang HS, Strain JF, Li Y, et al. West Nile virus neuroinvasive disease: neurological manifestations and prospective longitudinal outcomes. BMC Infect Dis (2014) 14:248. doi:10.1186/1471-2334-14-248

21. Huybrechts KF, Caro JJ. The Barthel index and modified Rankin scale as prognostic tools for long-term outcomes after stroke: a qualitative review of the literature. Curr Med Res Opin (2007) 23:1627-36. doi:10.1185/0300799 07X210444

22. Srihawan C, Castelblanco RL, Salazar L, Wootton SH, Aguilera E, OstroskyZeichner L, et al. Clinical characteristics and predictors of adverse outcome in adult and pediatric patients with healthcare-associated ventriculitis and meningitis. Open Forum Infect Dis (2016) 3:ofw077. doi:10.1093/ofid/ofw077

23. Randolph C, Tierney MC, Mohr E, Chase TN. The repeatable battery for the assessment of neuropsychological status (RBANS): preliminary clinical validity. J Clin Exp Neuropsychol (1998) 20:310-9. doi:10.1076/jcen.20.3. 310.823

24. Levin HS, O'Donnell VM, Grossman RG. The Galveston orientation and amnesia test. A practical scale to assess cognition after head injury. J Nerv Ment Dis (1979) 167:675-84. doi:10.1097/00005053-197911000-00004

25. Dale AM, Fischl B, Sereno MI. Cortical surface-based analysis. I. Segmentation and surface reconstruction. Neuroimage (1999) 9:179-94. doi:10.1006/ nimg.1998.0395

26. Fischl B, Sereno MI, Dale AM. Cortical surface-based analysis. II: inflation, flattening, and a surface-based coordinate system. Neuroimage (1999) 9:195-207. doi:10.1006/nimg.1998.0396

27. Ramasamy DP, Benedict RH, Cox JL, Fritz D, Abdelrahman N, Hussein S, et al. Extent of cerebellum, subcortical and cortical atrophy in patients with MS: a case-control study. J Neurol Sci (2009) 282:47-54. doi:10.1016/j.jns. 2008.12.034

28. Fischl B, Dale AM. Measuring the thickness of the human cerebral cortex from magnetic resonance images. Proc Natl Acad Sci U S A (2000) 97:11050-5. doi:10.1073/pnas.200033797

29. Avants BB, Epstein CL, Grossman M, Gee JC. Symmetric diffeomorphic image registration with cross-correlation: evaluating automated labeling of elderly and neurodegenerative brain. Med Image Anal (2008) 12:26-41. doi:10.1016/j. media.2007.06.004

30. Tao G, Datta S, He R, Nelson F, Wolinsky JS, Narayana PA. Deep gray matter atrophy in multiple sclerosis: a tensor based morphometry. J Neurol Sci (2009) 282:39-46. doi:10.1016/j.jns.2008.12.035

31. Datta S, Staewen TD, Cofield SS, Cutter GR, Lublin FD, Wolinsky JS, et al. Regional gray matter atrophy in relapsing remitting multiple sclerosis: baseline analysis of multi-center data. Mult Scler Relat Disord (2015) 4:124-36. doi:10.1016/j.msard.2015.01.004

32. Hobkirk AL, Starosta AJ, De Leo JA, Marra CM, Heaton RK, Earleywine M, et al. Psychometric validation of the BDI-II among HIV-positive CHARTER 
study participants. Psychol Assess (2015) 27:457-66. doi:10.1037/ pas0000040

33. Venkatesan A, Tunkel AR, Bloch KC, Lauring AS, Sejvar J, Bitnun A, et al. Case definitions, diagnostic algorithms, and priorities in encephalitis: consensus statement of the international encephalitis consortium. Clin Infect Dis (2013) 57:1114-28. doi:10.1093/cid/cit458

34. Govindarajan KA, Freeman L, Cai C, Rahbar MH, Narayana PA. Effect of intrinsic and extrinsic factors on global and regional cortical thickness. PLoS One (2014) 9(5):e96429. doi:10.1371/journal.pone.0096429

35. Natarajan N, Varman M. West Nile virus cerebellitis in a healthy 10-year-old child. Pediatr Infect Dis J (2007) 26:767. doi:10.1097/INF. 0b013e3180cc2c3f

36. Pruitt AA. Infections of the cerebellum. Neurol Clin (2014) 32:1117-31. doi:10.1016/j.ncl.2014.07.009

37. Jubelt B, Mihai C, Li TM, Veerapaneni P. Rhombencephalitis/brainstem encephalitis. Curr Neurol Neurosci Rep (2011) 11:543-52. doi:10.1007/ s11910-011-0228-5

38. Petropoulou KA, Gordon SM, Prayson RA, Ruggierri PM. West Nile virus meningoencephalitis: MR imaging findings. AJNR Am J Neuroradiol (2005) 26:1986-95.

39. DeQuesada I II, Saindane A. West Nile virus presenting as flaccid paralysis: case report and literature review. Radiol Case Rep (2012) 7:705. doi:10.2484/ rcr.v7i3.705
40. Beattie GC, Glaser CA, Sheriff H, Messenger S, Preas CP, Shahkarami M, et al. Encephalitis with thalamic and basal ganglia abnormalities: etiologies, neuroimaging, and potential role of respiratory viruses. Clin Infect Dis (2013) 56:825-32. doi:10.1093/cid/cis990

41. Carson PJ, Konewko P, Wold KS, Mariani P, Goli S, Bergloff P, et al. Longterm clinical and neuropsychological outcomes of West Nile virus infection. Clin Infect Dis (2006) 43:723-30. doi:10.1086/506939

42. Staples JE, Shankar MB, Sejvar JJ, Meltzer MI, Fischer M. Initial and long-term costs of patients hospitalized with West Nile virus disease. Am J Trop Med Hyg (2014) 90:402-9. doi:10.4269/ajtmh.13-0206

Conflict of Interest Statement: The authors declare that the research was conducted in the absence of any commercial or financial relationships that could be construed as a potential conflict of interest.

The reviewer BS and handling Editor declared their shared affiliation.

Copyright (c) 2018 Murray, Nolan, Ronca, Datta, Govindarajan, Narayana, Salazar, Woods and Hasbun. This is an open-access article distributed under the terms of the Creative Commons Attribution License (CC BY). The use, distribution or reproduction in other forums is permitted, provided the original author(s) and the copyright owner are credited and that the original publication in this journal is cited, in accordance with accepted academic practice. No use, distribution or reproduction is permitted which does not comply with these terms. 